\title{
Farmacogenética do tratamento da depressão: busca de marcadores moleculares de boa resposta aos
} antidepressivos

\author{
IVANOR Velloso Meira Lima ${ }^{1}$ \\ Everton Botelho SOUgeY ${ }^{1}$ \\ Homero Pinto Vallada Filho ${ }^{2}$
}

\begin{abstract}
Resumo
Os autores revisam os estudos que buscam determinantes genéticos para uma boa ou má resposta ao tratamento farmacológico da depressão, incluindo a verificação de diferenças interindividuais nos mecanismos farmacocinéticos - estudos de variantes gênicas das enzimas metabolizadoras das drogas - e farmacodinâmicos - estudos de variantes funcionais dos genes que codificam os sítios envolvidos no mecanismo de ação das drogas. Concluem que o conhecimento acerca da presença de variantes funcionais nos genes das isoformas envolvidas no metabolismo dos antidepressivos como o CYP2D6, ou nos genes codificando seus sítios de ação como a proteína transportadora de serotonina (5-HTT), podem fornecer importantes subsídios para a personalização da escolha de medicações e dosagens utilizadas no tratamento da depressão.
\end{abstract}

Palavras-chave: Antidepressivos, farmacogenética, polimorfismos.

\begin{abstract}
The present article reviews the studies on genetic determinants for drug response to the treatment of depression. These investigations assess the individual differences in the pharmacokinetic mechanisms - such as the genetic variants of genes coding for drug-metabolizing enzymes - and in the pharmacodynamics, looking at the genetic variants of genes coding for drug targets. The authors conclude that the knowledge about the functional variants of the genes coding for antidepressant metabolizing enzymes such as CYP2D6, or genes coding for antidepressant targets such as the serotonin transporter protein (5-HTT), could assist in individualizing the selection of medication and dosage for depression treatment.
\end{abstract}

Key Words: Antidepressant, pharmacogenetics, polymorphisms. Neuropsiquiatria da Universidade Federal de Pernambuco.

2 Laboratório de Neurociências (LIM 27), Departamento \& Instituto de Psiquiatria da Faculdade de Medicina da Universidade de São Paulo. 


\section{Introdução}

Desde 1950, consistente evidência tem sido obtida acerca da efetividade do tratamento farmacológico nas depressões. Os medicamentos antidepressivos têm reduzido a morbidade e melhorado o desfecho clínico de milhares de casos de depressão em todo o mundo.

Apesar do grande avanço nesta área e do desenvolvimento de novas classes de agentes antidepressivos, os profissionais envolvidos com o manejo desses pacientes ainda se defrontam com algumas dificuldades. Infelizmente cerca de 30\% a 40\% dos enfermos não respondem adequadamente ao tratamento inicial e podem levar cerca de seis semanas para caracterizar que um determinado medicamento não é efetivo para aquele caso (Doris et al., 1999).

Com isso, vários pesquisadores têm buscado identificar preditores clínicos eficientes para uma boa resposta ao fármaco a ser escolhido, o que poderia evitar a exposição do paciente a um longo período de terapia baseado na tentativa e erro, bem como reduziria a chance de grandes reações adversas.

Evidências oriundas de observações em famílias com múltiplos portadores de depressão apontam que fatores genéticos exercem relevante papel na resposta aos medicamentos antidepressivos ou na intensidade dos seus efeitos adversos (O'Reilly et al. 1994) e, nesse caminho, a farmacogenética surgiu como uma nova ferramenta diagnóstica que se propõe a buscar informações genéticas para subsidiar e guiar decisões da farmacoterapia e com isso melhorar o desfecho clínico com decisões clínicas personalizadas (Catalano, 1999).

Os estudos que buscam determinantes genéticos para uma boa ou má resposta ao tratamento farmacológico incluem a verificação de diferenças interindividuais nos mecanismos farmacocinéticos - estudos que focam variantes gênicas das enzimas metabolizadoras das drogas - e farmacodinâmicos - estudos que focam variantes funcionais dos genes que codificam os sítios envolvidos no mecanismo de ação das drogas.

\section{Estudos dos sítios relacionados com a farmacocinética dos antidepressivos}

Falhas na adesão ao tratamento com antidepressivos em geral decorrem do aparecimento de efeitos adversos em intensidade importante, e este é um aspecto relevante para a resposta ao fármaco.

Os antidepressivos são substratos do sistema enzimático Citocromo P450 em que existem várias isoformas enzimáticas codificadas por diferentes genes. Assim, variantes desses genes podem determinar enorme variabilidade na capacidade catalítica da enzima, podendo resultar em metabolizadores lentos com maior tendência a efeitos adversos ou tóxicos, metabolizadores normais e metabolizadores ultra-rápidos que também podem apresentar dificuldades na obtenção de concentrações plasmáticas adequadas para uma resposta terapêutica (Meyer, 2000).

As isoformas CYP2C19, CYP3A4 e CYP1A2 mediam a desmetilação de antidepressivos tricíclicos (metabolizam aminas terciárias como imipramina e amitriptilina para aminas secundárias farmacologicamente ativas como desipramina e nortriptilina), enquanto a isoforma CYP2D6 (hidroxilase de debrisoquina) é a principal envolvida na hidroxilação de tricíclicos e ISRS para metabólitos inativos.

Métodos baseados na reação em cadeia da polimerase - PCR já estão disponíveis para genotipagem dessas isoformas, e estudos populacionais demonstram que mutações em CYP2D6 causando decréscimo da atividade enzimática são encontradas em pelo menos $7 \%$ dos caucasianos que são pobres metabolizadores, portanto, candidatos a concentrações elevadas ou tóxicas de drogas antidepressivas com uso de doses convencionais. Por outro lado, duplicações do gene CYP2D6, resultando em tradução de maior quantidade da enzima, são encontrados em até 10\% dos caucasianos, produzindo metabolizadores ultra-rápidos que freqüentemente não conseguem obter concentrações adequadas com uso de doses habituais, e isto é particularmente relevante no uso de antidepressivos tricíclicos em que uma correlação entre a concentração da droga e os efeitos clínicos tem sido demonstrada (Steimer et al., 2001).

A maioria dos estudos realizados até o momento tem procurado relacionar o metabolismo dos antidepressivos tricíclicos e ISRS com genótipos das isoformas CYP2D6 e CYP2C19, sem que resultados inquestionáveis tenham sido estabelecidos pelas dificuldades no controle de variáveis confundidoras como idade, gênero e uso de comedicações (De Vries et al., 2002).

\section{Estudos de sítios relacionados com a farmacodinâmica dos antidepressivos}

Como a principal classe de antidepressivos utilizada atualmente é a dos medicamentos inibidores seletivos de recaptação de serotonina - ISRS, os principais marcadores biológicos estudados, até o presente, são 
componentes do sistema de transmissão serotoninérgico, incluindo-se enzimas de síntese, transportadores, receptores e metabolizadores desse neurotransmissor (Veenstra-Vander et al., 2000).

Admite-se que os ISRS interferem com a atividade da proteína que transporta a serotonina removendo-a da fenda sináptica, assim tem-se investigado variantes no gene do transportador de serotonina 5HTT.

Lesch et al., 1996 e Heils et al., 1997 descreveram um polimorfismo na região do promotor do gene 5HTT que consiste na presença ou ausência de 44 pares de bases, produzindo um alelo longo (L) e um curto (S). O alelo L associa-se a um aumento de atividade transcricional duas vezes maior que o alelo $\mathrm{S}$.

Desde então vários estudos têm mostrado associação do alelos $S$ com pobre resposta ao tratamento com ISRS. A Tabela 1 mostra os principais achados positivos com a variante do gene 5HTT.

$\mathrm{O}$ único resultado na direção oposta (Kim et al., 2000) foi observado numa amostra de asiáticos e pode dever-se à diferença significativa na freqüência do polimorfismo nessa população, comparada às populações ocidentais, e ao fato de o número de pacientes LL na amostra ser muito baixo $(\mathrm{N}=5)$. Além disso, neste estudo, houve uma taxa muito baixa de resposta dos pacientes que indiretamente confirma o papel positivo da variante LL com a resposta.

Outro candidato relacionado com resposta aos ISRS é o gene que codifica a enzima triptofano hidroxilase que é uma enzima que limita a biossítese da serotonina.

Dois estudos evidenciam associação da variante A779C (um potencial sítio de ligação de fatores de transcrição) com a resposta a ISRS:

Serreti et al. (2001a) relacionam o genótipo AA com pobre resposta à fluvoxamina numa amostra de $73 \mathrm{BP} e$ 144 DM ( $\mathrm{p}=0,001)$. Serreti et al. (2001b) relacionam também os genótipos AA e AC com pobre resposta à paroxetina numa amostra com $34 \mathrm{BP}$ e $87 \mathrm{DM}(\mathrm{p}=0,011)$.

Poucos estudos de farmacogenética analisam genes candidatos relacionados à farmacodinâmica das outras classes de antidepressivos (tricíclicos, IMAOs etc.)
Entre os candidatos estudados, destacamos variantes do gene da MAO-A, principal enzima de degradação das aminas biogênicas, mutações na proteína de ligação do nucleotídio guanina - proteína G $\beta 3$ - relacionadas com processos regulatórios transmembrana de muitos receptores do SNC, e a variação gênica C102T do receptor pós-sináptico 5HT2A. A Tabela 2 apresenta os principais estudos nesta linha.

\section{Conclusão}

O conhecimento acerca da presença ou não de mutações nos genes das isoformas envolvidas no metabolismo dos antidepressivos ou nos genes codificados em seus sítios de ação podem fornecer subsídios importantes para ajudar no manejo de dosagens e personalização da escolha das medicações utilizadas para o tratamento da depressão.

Contudo, no presente, a aplicabilidade clínica desses conhecimentos carece ainda de consideráveis investimentos na obtenção de dados oriundos de estudos prospectivos que permitam uma correlação consistente das informações obtidas com a genotipagem de variantes, relacionadas à farmacodinâmica e farmacocinética dos agentes antidepressivos, com fenótipos de resposta terapêutica a esses agentes em diferentes grupos populacionais.

Por fim, é importante ressaltar que os estudos acerca dos mecanismos moleculares envolvidos na resposta aos antidepressivos podem ainda ser uma fonte de informações importantes para o entendimento da própria neurobiologia da depressão.

Tabela 1: Variantes do gene 5 HTT e resposta aos ISRS

\begin{tabular}{lccc}
\hline Autor & Amostra & Droga & Resultado \\
Smeraldi et al., 1998 & $30 \mathrm{BP}$ e $69 \mathrm{DM}$ & Fluvoxamina & Alelo $\mathrm{L} /$ boa resposta $(\mathrm{p}=0,001)$ \\
Zanardi et al., 2001 & $47 \mathrm{BP}$ e $108 \mathrm{DM}$ & Fluvoxamina & Alelo $\mathrm{L}$ boa resposta $(\mathrm{p}=0,029)$ \\
Zanardi et al., 2000 & $18 \mathrm{BP}$ e 46 DM & Paroxetina & Alelo $\mathrm{S} / \mathrm{má} \mathrm{resposta}(\mathrm{p}<0,001)$ \\
Pollock et al., 2000 & $95 \mathrm{Dep}$ tardia & Paroxetina & Alelo $\mathrm{S} / \mathrm{má} \mathrm{resposta}(\mathrm{p}=0,028)$ \\
Kim et al., 2000 & $120 \mathrm{DM}$ coreanos & Fluoxetina e paroxetina & Genótipo $\mathrm{SS} /$ boa resposta $(\mathrm{p}=0,007)$ \\
Arial et al., 2001 & $102 \mathrm{DM}$ & Citalopram & Genótipo $\mathrm{SS} /$ grupo sem remissão $(\mathrm{p}=0,006)$
\end{tabular}

$\mathrm{BP}=$ bipolar; $\mathrm{DM}=$ Depressão maior 
Tabela 2: Outras variantes gênicas estudadas em diferentes classes de antidepressivos

$\begin{array}{lcccc}\text { Autor } & \text { Gene } & \text { Amostra } & \text { Droga } & \text { Resultado } \\ \text { Muller et al., } 2000 & \text { MAO-A } & 64 \mathrm{DN} & \text { Moclobemida } \\ \text { Zill et al., } 2000 & \text { Proteina G } \beta 3 & 10 \text { BP e 78 DM } & \text { Tricíclicos e ISRS } & \text { Genótipo TT/ boa resposta (p = 0,01) } \\ \text { Minov et al., } 2001 & 5 \mathrm{HT} 2 \mathrm{~A} & 173 \mathrm{DM} & \text { Tricíclicos e ISRS } & \text { Alelo c/ boa resposta (p = 0,023) }\end{array}$

\section{Referências bibliográficas}

Catalano, M. - The challenges of psychopharmacogenetics. Am. J. Hum. Genet. 65: 606-10, 1999.

Denney, R.M.; Koch, H.; Craig, I.W. - Association between monoamine oxidase A activity in human male skin fibroblasts and genotype of the MAO-A promoter-associated variable number tandem repeat. Hum. Genet 105: 542-51, 1999.

Doris, A.; Ebmeier, K.; Shajahan, P. - Depressive Illness. Lancet 354: 1369$75,1999$.

FANG, J.; GorroD, J.W. - Metabolism, pharmacogenetics, and metabolic drug-drug interactions of antipsychotic drugs. Cell. Mol. Neurobiol. 19: 491-510 1999.

Heils, A.; Teufel, A.; Petri, S.; Stöber, G.; Riederer, P.; Bengel, D.; Lesch, P. Allelic variation of human serotonin transporter gene expression. J. Neurochem. 66: 2621-24 1996.

Kim, D.K.; Lim, S.W.; LeE, S.; Sohn, S.E.; Kim, S.; Hahn, C.G.; Carroll, B.J. Serotonin transporter gene polymorphism and antidepressant response. NeuroReport 11: 215-19, 2000.

Lesch, K.P.; Bengel, D.; Heils, A.; Sabol, S.; Greenberg, B.; Petri, S.; Benjamin, J.; Muller, C.; Hamer, D.; Murphy, D. - Association of anxiety-related traits with a polymorphism the in serotonin transporter gene regulatory region. Science 274: 1527-30, 1996.

MeYer, U.A. - Pharmacogenetics and adverse drug reactions. Lancet 356: $1667-71,2000$.

Muller, D.J.; Schulze, T.G.; Macciardi, F.; Ohlraun, S.; Gross, M.M.; Bauer, I.; Scherk, H.; KischKel, E.; Neidt, H.; Syagailo, Y.V.; Grassle, M.; Nothen, M.M.; Maier, W.; Lesch, K.P.; Rietschel, M. - Moclobemide response in depressed patients: association study with a functional polymorphism in the monoamine oxidase-A promoter. Neuropsychiatric Genetics 96 (4). (2000): 537.

Woo-Ming, A.; Mitropoulou, V.; Silverman, J.; Reynolds, D.; Schopick, F.; TrestMan, R.L.; Siever, L.J. - Serotonin and the prediction of response time to fluoxetine in patients with mild depression. Psychiatry Res. 88: 89-93, 1999.

Nielsen, D.A.; Jenkins, G.L.; Stefanisko, K.M.; Jefferson, K.K.; Goldman, D. Sequence, splice site and population frequency distribution analyses of the polymorphic human tryptophan hydroxylase intron 7. Mol. Brain Res. 45: 145-8, 1997.

O’Reilly, R.L.; Bogue, L.; Singh, S.M. - Pharmacogenetic response to antidepressants in a multicase family with affective disorder. Biol. Psychiatry 36: 467-71, 1994.

PICKAR, D.; Rubinow, R. - Pharmacogenomics of psychiatric disorders.
Trends Pharmacol. Sci. 22: 75-83, 2001.

Pollock, B.G.; Ferrell, R.E.; Mulsant, B.H.; Mazumdar, S.; Miller, M.; Sweet R.A.; DAvis, S.; KIRShNer, S.A.; HoucK, P.R.; STACK, J.A.; ReynoldS, C.F.; KuPFER, D.J. - Allelic variation in the serotonin transporter promoter affects onset of paroxetine treatment response in late-life depression. Neuropsychopharmacology 23: 587-90, 2000.

Sabol, S.Z.; Hu, S.; Hamer, D. - A functional polymorphism in the monoamine oxidase A gene promoter. Hum. Genet. 103: 273-9, 1998.

SCHAFER, W.F. - How do antidepressants work? Prospects for genetic analysis of drug mechanisms. Cell 98: 551-4, 1999.

Schloss, P.; Williams, D.C. - The serotonin transporter a primary target for antidepressant drugs. J. Psychopharmacol. 12: 115-21, 1998.

Serretti, A.; Zanardi, R.; Cusin, C.; Rossini, D.; Lorenzi, C.; Smeraldi, E. - Tryptophan hydroxylase gene associated with paroxetine antidepressant activity. Eur. Neuropsychopharmacol. 11: 375-80, 2001 a.

Serretti, A.; Zanardi, R.; Rossini, D.; Cusin, D.; Lilli, R.; Smeraldi, E. Influence of tryptophan hydroxylase and serotonin transporter genes on fluvoxamine antidepressant activity. Mol. Psychiatry 6: 586-92, 2001b.

Smeraldi, R.; Zanardi, F.; Benedetti, D.; Dibella, J.; Perez J.; Catalano, M. Polymorphism within the promoter of the serotonin transporter gene and antidepressant efficacy of fluvoxamine. Mol. Psychiatry 3: 508-11, 1998.

Steimer, W.; Müller, B.; Leuchet, S.; Kisssling, W. - Pharmacogenetics: A new diagnostic tool in the management of antidepressive drug therapy. Clin Chim Acta 308: 33-41, 2001.

Veenstra-Vander Weele, J.; Anderson, G.M.; CoOK, E.H. - Pharmacogenetics and the serotonin system: initial studies and future directions. Eur. J. Pharmacol. 410: 165-81, 2000.

Whale, R.; Quested, D.J.; Laver, D.; Harrison, P.J.; Cowen, P.J. - Serotonin transporter (5-HTT) promoter genotype may influence the prolactin response to clomipramine. Psychopharmacology (Berlin) 150: 120-2, 2000.

Zanardi, R.; Benedetti, F.; DiBella, D.; Catalano, M.; Smeraldi, E. - Efficacy of paroxetine in depression is influenced by a functional polymorphism within the promoter of serotonin transporter gene. J. Clin. Psychopharmacol. 20: 105-7, 2000.

Zill, P.; Baghai, T.C.; Zwanzger, P.; Schule, C.; Minov, C.; Riedel, M.; Neumeier, K.; Rupprecht, R.; Bondy, B. - Evidence for an association between a G-protein beta3-gene variant with depression and response to antidepressant treatment. NeuroReport 11: 1893-97, 2000. 